\title{
Anfíbios anuros associados a corpos d'água do sudoeste do estado de Goiás, Brasil
}

\author{
Alessandro Ribeiro Morais ${ }^{1,6}$, Luciana Signorelli ${ }^{1}$, Priscilla Guedes Gambale ${ }^{1}$, \\ Kátia Kopp², Fausto Nomura ${ }^{1,3}$, Lorena Dall'ara Guimarães ${ }^{4}$, Wilian Vaz-Silva ${ }^{5}$, \\ Jade Ramos ${ }^{1}$ \& Rogério Pereira Bastos ${ }^{1,3}$ \\ ${ }^{1}$ Laboratório de Herpetologia, Instituto de Ciências Biológicas, Universidade Federal de Goiás - UFG, \\ CP 131, CEP 74001-970, Goiânia, GO, Brasil \\ ${ }^{2}$ Escola de Engenharia Civil, Universidade Federal de Goiás - UFG, CEP 74605-220, Goiânia, GO, Brasil \\ ${ }^{3}$ Departamento de Ecologia, Instituto de Ciências Biológicas, Universidade Federal de Goiás - UFG, \\ CP 131, CEP 74001-970, Goiânia, GO, Brasil \\ ${ }^{4}$ Centro de Ensino e Pesquisa Aplicada à Educação, Universidade Federal de Goiás - UFG, \\ CP 131, CEP 74001-970, Goiânia, GO, Brasil \\ ${ }^{5}$ Departamento de Ciências Biológicas, Centro Universitário de Goiás - Uni-Anhanguera, \\ Rua Professor Lázaro Costa, 456, CEP 74415-450, Goiânia, GO, Brasil \\ ${ }^{6}$ Autor para correspondência: Alessandro Ribeiro Morais, e-mail: alessandrogyn@ hotmail.com
}

MORAIS, A.R., SIGNORELli, L., GAMBALE, P.G., KOPP, K., NOMURA, F., GUIMARÃES, L.D., VAZ-SILVA, W., RAMOS, J. \& BASTOS, R.P. Anuran amphibians associated to water bodies in Southwest of Goiás State (Brazil). Biota Neotrop. 11(3): http://www.biotaneotropica.org.br/v11n3/en/abstract?inventory + bn03611032011

Abstract: The Cerrado is the second largest biome in Brazil and is considered one of most important among the biodiversity hotspots. In this biome can be found about 150 anurans species, from which $30 \%$ are endemic. However, there are many Cerrado areas that have little information gathered about the anuran biodiversity. Thus, in this study we present the result of an inventory of anurans species from the southwest region of Goiás State (Central Brazil). In this region, we sampled 45 water bodies, located near soybean and sugar-cane cultures, pastures and natural vegetation, from November/2007 to February/2008 and from November/2008 to March/2009. Altogether, adults and tadpoles of 36 species were registered, with the majority of species registered in association to open areas. The rarefied curve of species richness show an asymptote formation, which indicates that the chance to add species to the inventory is low, even with additional habitats sampling. Therefore, our results reveal high species richness in the present sampled region, which represented almost one fourth of all species found in Cerrado. Keywords: Brazilian savanna, species distribution, central Brazil.

MORAIS, A.R., SIGNORELli, L., GAMBALE, P.G., KOPP, K., NOMURA, F., GUIMARÃES, L.D., VAZ-SILVA, W., RAMOS, J. \& BASTOS, R.P. Anfíbios anuros associados a corpos d'água do sudoeste do estado de Goiás (Brasil). Biota Neotrop. 11(3): http://www.biotaneotropica.org.br/v11n3/pt/abstract?inve ntory+bn03611032011

Resumo: O Cerrado é o segundo maior bioma brasileiro e é considerado um dos mais importantes hotspots de biodiversidade. Neste bioma foram registradas cerca de 150 espécies de anuros, das quais $30 \%$ são endêmicas. Entretanto, existem muitas áreas ainda não inventariadas, nas quais não se conhece quase nada sobre a diversidade de anuros. Assim, neste estudo apresentamos informações referentes a anurofauna do sudoeste do Estado de Goiás (Brasil Central), a qual vem sendo modificada principalmente pela agropecuária. Nesta região, 45 corpos d'água foram amostrados entre os meses de novembro/2007 a fevereiro/2008 e novembro/2008 a março/2009, localizados próximos a plantações de soja, cana-de-açúcar, pastagem e vegetação nativa. No total, foram registrados adultos e girinos de 36 espécies de anuros, a maioria comum de áreas abertas. As curvas de acumulação de espécies, tanto para adultos quanto para girinos, apresentaram a formação de uma assíntota, o que indica uma baixa probabilidade de registro de novas espécies mesmo com a amostragem de outros corpos d'água. Nesta área com forte influência de impactos agrícolas, a riqueza encontrada representa aproximadamente 1/4 do total de espécies conhecidas para o bioma Cerrado.

Palavras-chaves: cerrado, distribuição de espécies, Brasil central. 


\section{Introdução}

Mittermeier et al. (1998) e Myers et al. (2000) consideraram o Cerrado como um dos 25 Hotspots do planeta, no qual se observa uma alta diversidade de espécies endêmicas submetidas a uma excepcional perda de habitat, devido ao alto grau de degradação ou modificação ambiental. Este bioma, ao longo dos últimos 40 anos, tem sofrido intensa ação antrópica devido aos diferentes tipos de uso do solo (Klink \& Machado 2005). Assim, o Cerrado sofre com uma elevada perda de biodiversidade, inclusive de espécies com distribuição restrita ou com pequeno tamanho corporal que ainda não foram descritas formalmente (Diniz-Filho et al. 2005, 2009).

Historicamente, a maioria dos estudos sobre anfíbios foram realizados ao longo do litoral ou de grandes rios (Brandão \& Araújo 1998), enquanto que apenas recentemente as assembléias de anfíbios de regiões interioranas têm sido estudadas (e.g. Rossa-Feres \& Jim 2001, Bastos et al. 2003, Giaretta et al. 2008). O Cerrado apresenta uma elevada diversidade de anfíbios, sendo conhecidas cerca de 150 espécies, ou aproximadamente $20 \%$ do total encontrado no Brasil (Colli et al. 2002, Bastos 2007). Deste total, aproximadamente 47 espécies são endêmicas do Cerrado, enquanto outras possuem ampla distribuição, ocorrendo também em áreas de Mata Atlântica, Amazônia e Caatinga (Bastos 2007).

Os estudos realizados na região do Cerrado incluem trabalhos sobre história natural (Bastos et al. 2003, Guimarães \& Bastos 2003, Martins \& Jim 2003, Alcântara et al. 2007), e sobre estrutura de comunidade (Eterovick \& Sazima 2000, Diniz-Filho et al. 2004a, b, Giaretta et al. 2008). Mesmo assim, informações sobre os padrões de diversidade, uso de habitat e distribuição dessas espécies ainda são escassos, existindo inúmeras localidades não amostradas ou com informações insuficientes sobre as espécies (Colli et al. 2002, Bastos 2007).

Neste trabalho apresentamos o resultado do inventariamento das espécies de anfíbios anuros, tanto na fase adulta quanto larval, em uma área de intensa atividade agropastoril do sudoeste do estado de Goiás (Brasil Central). Com isso, além de contribuir para o conhecimento da biodiversidade, apresentamos informações sobre a distribuição das espécies que podem servir como subsídio para políticas públicas de conservação e ações de manejo de espécies.

\section{Material e Métodos}

O estudo foi realizado em 45 corpos d'água (Figura 1, Tabela 1), em seis municípios: Rio Verde

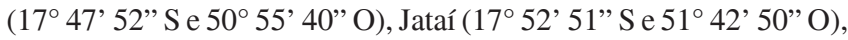
Serranópolis $\left(18^{\circ} 18^{\prime} 21^{\prime \prime} \mathrm{S}\right.$ e $\left.51^{\circ} 57^{\prime} 43^{\prime \prime} \mathrm{O}\right)$, Mineiros (17 $34^{\circ} 8^{\prime \prime} \mathrm{S}$ e $52^{\circ} 33^{\prime}$ ' "' O), Chapadão do Céu ( $18^{\circ} 23^{\prime} 34^{\prime \prime}$ S e $\left.52^{\circ} 39^{\prime} 57^{\prime \prime} \mathrm{O}\right)$ e Portelândia $\left(17^{\circ} 21^{\prime} 30.02^{\prime \prime} \mathrm{S}\right.$ e $52^{\circ} 40^{\prime} 32.4$ " O). A região apresenta diversas fitofisionomias de Cerrado variando de formações abertas, como campo limpo e campo rupestre, a formações mais densas, tais como Cerradão e Florestas Estacionais Semideciduais (Valente 2006). O clima é tropical quente e úmido, apresentando duas estações bem definidas, sendo uma seca de abril a setembro e outra chuvosa de outubro a março (Miner 1989). Os corpos d'água amostrados incluíam brejos, poças permanentes e temporárias, localizados próximos a plantações de soja, cana-de-açúcar, pastagem ou vegetação nativa.

As coletas foram realizadas ao longo das estações chuvosas de novembro/2007 - fevereiro/2008 e novembro/2008 - março/2009. Neste período foram realizadas nove visitas a campo, perfazendo um total de 35 dias de amostragem, com cada ponto amostral sendo visitado somente uma vez. Para os anuros em fase adulta, os corpos d'água foram amostrados durante o período noturno utilizando-se os métodos de "amostragem por encontro visual" (Crump \& Scott Junior 1994) e "transectos auditivos" (Zimmerman 1994). Para melhor padronização dos dados, os corpos d'água foram percorridos durante o período de uma hora. Alguns indivíduos tiveram suas vocalizações gravadas com auxílio de gravador MARANTZ PMD 222 acoplado a um microfone direcional Sennheiser ME66 para posterior confirmação da identidade da espécie através dos atributos temporais e espectrais do canto de anúncio.

Indivíduos testemunhos foram coletados, anestesiados com xilocaína a $5 \%$, fixados em solução de formalina a 10\% e, posteriormente, conservados em álcool a 70\%. Quanto aos girinos, as amostragens foram realizadas com puçá de tela de arame de $3 \mathrm{~mm}^{2}$, passados nas margens e no interior dos ambientes estudados, durante uma hora. Os girinos foram coletados, anestesiados em solução de mentol, e fixados em formalina a $10 \%$ (Heyer et al. 1994). A identificação foi feita com base em Pugliesi et al. (2001), Brandão (2002) e Rossa-Feres \& Nomura (2006) utilizando-se estereomicroscópio Zeiss. Os espécimes testemunho estão depositados na Coleção Zoológica da Universidade Federal de Goiás (ZUFG).

Considerando que a riqueza de espécies observadas é freqüentemente um estimador viciado em relação à riqueza de espécies real (Santos 2006), foi usado o estimador não paramétrico Jackknife de $1^{\mathrm{a}}$ ordem para extrapolação da riqueza de espécies na região (Coddington et al. 1991, Colwell \& Coddington 1994), utilizando cada poça como unidade amostral. Este estimador extrapola a riqueza observada pela frequiência de espécies raras ("uniques") presentes nas amostras. Essa técnica produz uma estimativa mais aproximada da riqueza de uma comunidade (Krebs 1999), fornecendo ainda um intervalo de confiança que permite a realização de comparações estatísticas entre duas ou mais localidades amostradas. Além disso, este método é considerado como o mais preciso e acurado quando comparado a outros métodos de estimativa de riqueza de espécies (Walther \& Morand 1998).

\section{Resultados}

Foram registradas 36 espécies de anfíbios anuros pertencentes a seis famílias e 12 gêneros (Figura 2, Tabela 2). Nenhuma das espécies registradas é considerada ameaçada de acordo com as listas vermelhas da IUCN (International... 2010) e do Brasil (Haddad 2008). Quanto à representatividade das famílias, Hylidae (18 spp.) apresentou o maior número de espécies, seguida pelas famílias Leptodactylidae (oito spp.), Leiuperidae (cinco spp.) e Bufonidae (duas spp.). As famílias Cycloramphidae e Microhylidae foram representadas por uma espécie cada.

Deste total de espécies, 35 foram registradas apenas na fase adulta (Tabela 2) das quais 27 espécies (71\% do total) foram registradas também na fase de girino (Tabela 2). Considerando o estágio de vida, o número estimado de espécies para a área variou de 43,84 \pm 8,19, relativo apenas aos adultos, e 33,84 $\pm 6,08$, levando-se em consideração somente os girinos. Para ambas as fases de vida, a curva do coletor apresentou tendência à estabilização, pois os intervalos de confiança da riqueza estimada e observada (Figura 3) se sobrepõem consideravelmente, não sendo estatisticamente diferentes entre si. Todavia para a fase adulta, houve maior sobreposição entre estes.

As espécies mais comuns foram Physalaemus cuvieri e Scinax fuscomarginatus, registradas em 66 e $60 \%$ dos locais de amostragem, respectivamente. Somente cinco espécies (Dendropsophus soaresi, Leptodactylus syphax, Odontophrynus aff. americanus, Phyllomedusa sp. (gr. hypochondrialis) e Scinax $x$-signatus) foram encontradas em um único ponto de coleta. Hypsiboas lundii, Phyllomedusa sp. (gr. hypochondrialis) e Leptodactylus syphax foram coletadas apenas em corpos d'água próximos a ambientes florestais ou de afloramentos rochosos, enquanto que as demais espécies foram encontradas em áreas abertas. 
Anuros do sudoeste goiano

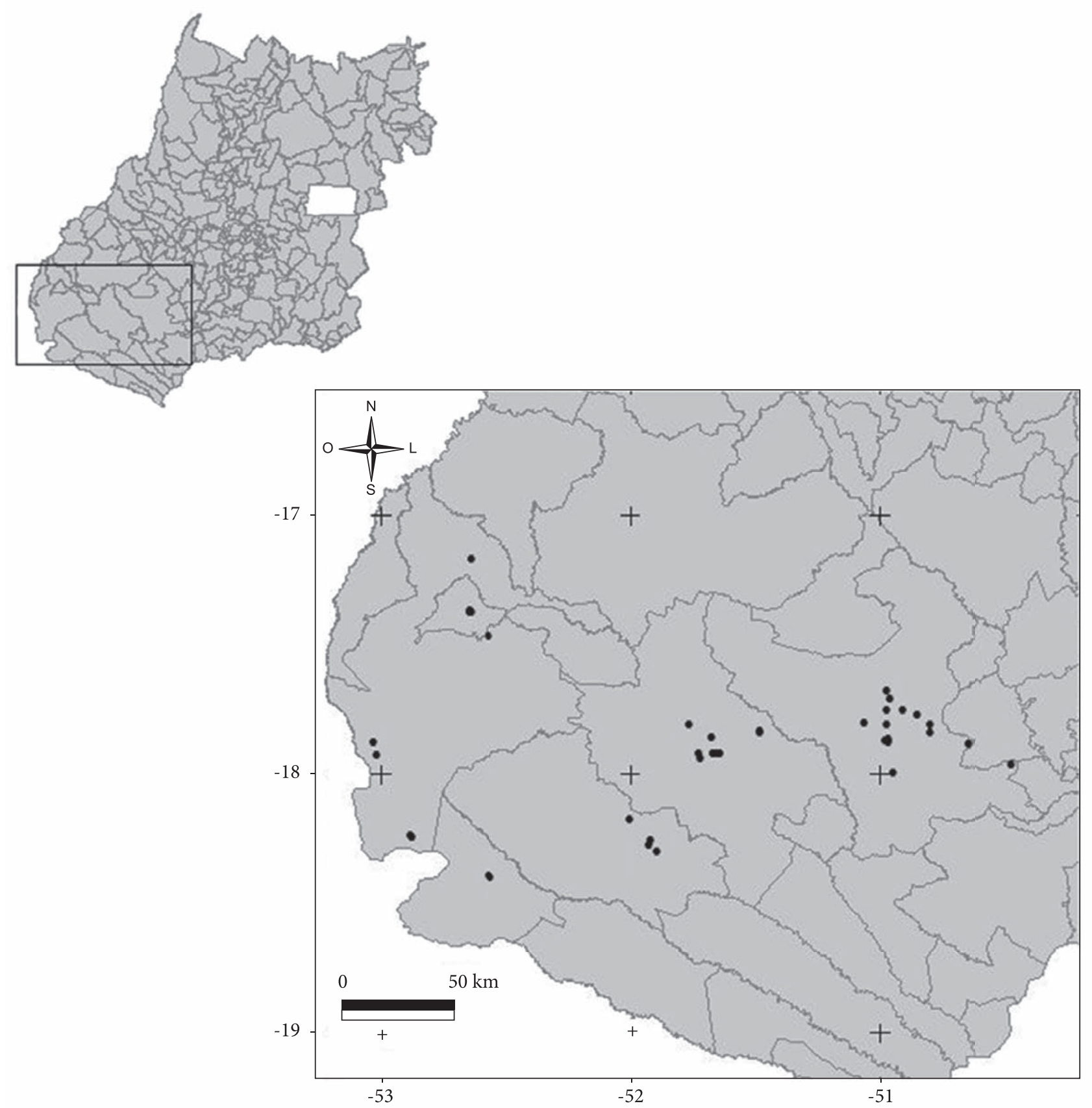

Figura 1. Localização dos corpos d’água amostrados na região sudoeste, estado de Goiás, Brasil.

Figure 1. Location of water bodies sampled in southwest region, Goiás state, Brazil.

Tabela 1. Lista dos corpos d’água, incluíndo características físicas, amostrados no sudoeste do estado de Goiás, Brasil.

Table 1. list of water bodies, including physical characteristics, sampled in the southwestern state of Goiás, Brazil.

\begin{tabular}{|c|c|c|c|c|c|c|}
\hline \multirow[t]{2}{*}{ Corpos d’água } & \multicolumn{2}{|c|}{ Coordenadas } & \multirow[t]{2}{*}{ Altitude } & \multirow[t]{2}{*}{ Tamanho* } & \multirow{2}{*}{$\begin{array}{l}\text { Tipo de fragmento } \\
\text { próximo }\end{array}$} & \multirow[t]{2}{*}{ Tipo de vegetação na margem } \\
\hline & Latitude & Longitude & & & & \\
\hline 1 & $18^{\circ} 11^{\prime} 07^{\prime \prime}$ & $52^{\circ} 01^{\prime} 04^{\prime \prime}$ & 790 & Médio & Mata e pastagem & Arbustiva e arbórea \\
\hline 2 & $18^{\circ} 11^{\prime} 24^{\prime \prime}$ & $52^{\circ} 01^{\prime} 51^{\prime \prime}$ & 760 & Pequeno & Mata e pastagem & Herbácea, arbustiva e arbórea \\
\hline 3 & $17^{\circ} 59^{\prime} 55^{\prime \prime}$ & $50^{\circ} 58^{\prime} 10^{\prime \prime}$ & 665 & Pequeno & Plantação & Herbácea \\
\hline 4 & $17^{\circ} 48^{\prime} 06^{\prime \prime}$ & $51^{\circ} 05^{\prime} 22^{\prime \prime}$ & 800 & Grande & Mata e pastagem & Herbácea e arbustiva \\
\hline 5 & $17^{\circ} 48^{\prime} 35.2^{\prime \prime}$ & $50^{\circ} 59^{\prime} 50.6^{\prime \prime}$ & 780 & Médio & Mata e plantação & Herbácea \\
\hline 6 & $18^{\circ} 25^{\prime} 32^{\prime \prime}$ & $52^{\circ} 35^{\prime} 24^{\prime \prime}$ & 770 & Médio & Mata e plantação & Herbácea e arbustiva \\
\hline
\end{tabular}

*Pequeno < $500 \mathrm{~m}^{2}$; Média: entre $500 \mathrm{~m}^{2}$ e $2000 \mathrm{~m}^{2}$; Grande $>2000 \mathrm{~m}^{2}$.

$*$ Small < $500 \mathrm{~m}^{2}$; Average: entre $500 \mathrm{~m}^{2}$ e $2000 \mathrm{~m}^{2}$; Great $>2000 \mathrm{~m}^{2}$. 
Morais, A.R. et al.

Tabela 1. Continuação...

\begin{tabular}{|c|c|c|c|c|c|c|}
\hline \multirow[t]{2}{*}{ Corpos d’água } & \multicolumn{2}{|c|}{ Coordenadas } & \multirow[t]{2}{*}{ Altitude } & \multirow[t]{2}{*}{ Tamanho* } & \multirow{2}{*}{$\begin{array}{c}\text { Tipo de fragmento } \\
\text { próximo }\end{array}$} & \multirow[t]{2}{*}{ Tipo de vegetação na margem } \\
\hline & Latitude & Longitude & & & & \\
\hline 7 & $17^{\circ} 48^{\prime} 34.2^{\prime \prime}$ & $50^{\circ} 49^{\prime} 17.9^{\prime \prime}$ & 665 & Pequeno & Plantação & Sem vegetação \\
\hline 8 & $17^{\circ} 55^{\prime} 41^{\prime \prime}$ & $53^{\circ} 02^{\prime} 41^{\prime \prime}$ & 820 & Grande & Mata e plantação & Herbácea \\
\hline 9 & $17^{\circ} 45^{\prime} 2.1^{\prime \prime}$ & $50^{\circ} 56^{\prime} 7.4^{\prime \prime}$ & 750 & Grande & Mata e pastagem & Herbácea e arbustiva \\
\hline 10 & $18^{\circ} 16^{\prime} 21.1^{\prime \prime}$ & $51^{\circ} 56^{\prime} 35.4^{\prime \prime}$ & 745 & Grande & Mata e pastagem & Herbácea, arbustiva e arbórea \\
\hline 11 & $17^{\circ} 42^{\prime} 20.4^{\prime \prime}$ & $50^{\circ} 59^{\prime} 0.3{ }^{\prime \prime}$ & 785 & Médio & Pastagem & Herbácea, arbustiva e arbórea \\
\hline 12 & $17^{\circ} 51^{\prime} 57.5^{\prime \prime}$ & $50^{\circ} 59^{\prime} 24.4^{\prime \prime}$ & 680 & Médio & Mata e pastagem & Herbácea \\
\hline 13 & $17^{\circ} 52^{\prime} 36.2^{\prime \prime}$ & $50^{\circ} 59^{\prime} 35.9^{\prime \prime}$ & 680 & Grande & Mata e pastagem & Herbácea, arbustiva e arbórea \\
\hline 14 & $17^{\circ} 52^{\prime} 31.2^{\prime \prime}$ & $50^{\circ} 59^{\prime} 33.8^{\prime \prime}$ & 690 & Médio & Mata e plantação & Herbácea, arbustiva e arbórea \\
\hline 15 & $17^{\circ} 52^{\prime} 13.3^{\prime \prime}$ & $51^{\circ} 00^{\prime} 7.1^{\prime \prime}$ & 715 & Médio & Mata e plantação & Herbácea, arbustiva e arbórea \\
\hline 16 & $17^{\circ} 21^{\prime} 18.6^{\prime \prime}$ & $52^{\circ} 40^{\prime} 12.6^{\prime \prime}$ & 830 & Pequeno & Mata & Herbácea e arbustiva \\
\hline 17 & $17^{\circ} 21^{\prime} 17.2^{\prime \prime}$ & $52^{\circ} 40^{\prime} 9.0^{\prime \prime}$ & 850 & Médio & Mata & Herbácea, arbustiva e arbórea \\
\hline 18 & $18^{\circ} 17^{\prime} 39.2^{\prime \prime}$ & $51^{\circ} 56^{\prime} 58.5^{\prime \prime}$ & 840 & Grande & Mata & Herbácea, arbustiva e arbórea \\
\hline 19 & $17^{\circ} 21^{\prime} 6.4^{\prime \prime}$ & $52^{\circ} 40^{\prime} 26.6^{\prime \prime}$ & 840 & Grande & Mata & Herbácea, arbustiva e arbórea \\
\hline 20 & $17^{\circ} 21^{\prime} 4.4^{\prime \prime}$ & $52^{\circ} 40^{\prime} 24.8^{\prime \prime}$ & 830 & Médio & Mata & Herbácea, arbustiva e arbórea \\
\hline 21 & $18^{\circ} 19^{\prime} 8.3^{\prime \prime}$ & $51^{\circ} 55^{\prime} 12^{\prime}$ & 675 & Médio & Plantação & Herbácea e arbustiva \\
\hline 22 & $17^{\circ} 52^{\prime} 49^{\prime \prime}$ & $53^{\circ} 03^{\prime} 36^{\prime \prime}$ & 795 & Médio & Plantação & Herbácea e arbustiva \\
\hline 23 & $18^{\circ} 24^{\prime} 45^{\prime \prime}$ & $52^{\circ} 34^{\prime} 55^{\prime \prime}$ & 755 & Médio & Mata e plantação & Herbácea, arbustiva e arbórea \\
\hline 24 & $17^{\circ} 53^{\prime} 02^{\prime}$ & $50^{\circ} 40^{\prime} 23^{\prime \prime}$ & 570 & Médio & Mata e plantação & Herbácea, arbustiva e arbórea \\
\hline 25 & $17^{\circ} 50^{\prime} 36^{\prime \prime}$ & $50^{\circ} 49^{\prime} 21,5^{\prime \prime}$ & 690 & Grande & Plantação & Herbácea e arbustiva \\
\hline 26 & $17^{\circ} 46^{\prime} 17^{\prime \prime}$ & $50^{\circ} 52^{\prime} 36.3^{\prime \prime}$ & 715 & Grande & Mata e plantação & Herbácea, arbustiva e arbórea \\
\hline 27 & $17^{\circ} 45^{\prime} 01^{\prime \prime}$ & $50^{\circ} 52^{\prime} 49^{\prime \prime}$ & 690 & Grande & Mata e plantação & Herbácea, arbustiva e arbórea \\
\hline 28 & $17^{\circ} 27^{\prime} 20.4^{\prime \prime}$ & $52^{\circ} 35^{\prime} 49.7^{\prime \prime}$ & 850 & Grande & Mata e pastagem & Herbácea, arbustiva e arbórea \\
\hline 29 & $17^{\circ} 55^{\prime} 26^{\prime \prime}$ & $51^{\circ} 45^{\prime} 10^{\prime \prime}$ & 740 & Pequeno & Mata e plantação & Herbácea e arbustiva \\
\hline 30 & $17^{\circ} 56^{\prime} 43^{\prime \prime}$ & $51^{\circ} 44^{\prime} 39^{\prime \prime}$ & 760 & Pequeno & Mata e plantação & Herbácea e arbustiva \\
\hline 31 & $17^{\circ} 51^{\prime} 24.6^{\prime \prime}$ & $51^{\circ} 41^{\prime} 54.4^{\prime \prime}$ & 660 & Pequeno & Mata e plantação & Arbustiva \\
\hline 32 & $17^{\circ} 55^{\prime} 31.6^{\prime \prime}$ & $51^{\circ} 41^{\prime} 31.7^{\prime \prime}$ & 675 & Pequeno & Mata & Herbácea e arbustiva \\
\hline 33 & $17^{\circ} 55^{\prime} 28.9^{\prime \prime}$ & $51^{\circ} 41^{\prime} 27.7^{\prime \prime}$ & 740 & Pequeno & Mata e plantação & Herbácea e arbustiva \\
\hline 34 & $17^{\circ} 55^{\prime} 36.6^{\prime \prime}$ & $51^{\circ} 41^{\prime} 35.8^{\prime \prime}$ & 740 & Pequeno & Mata & Herbácea e arbustiva \\
\hline 35 & $17^{\circ} 55^{\prime} 38.3^{\prime \prime}$ & $51^{\circ} 40^{\prime} 8.8^{\prime \prime}$ & 690 & Médio & Plantação & Sem vegetação \\
\hline 36 & $17^{\circ} 55^{\prime} 32.4^{\prime \prime}$ & $51^{\circ} 40^{\prime} 4.2^{\prime \prime}$ & 680 & Pequeno & Plantação & Sem vegetação \\
\hline 37 & $17^{\circ} 50^{\prime} 11.6^{\prime \prime}$ & $51^{\circ} 30^{\prime} 22^{\prime \prime}$ & 825 & Médio & Mata e plantação & Arbustiva \\
\hline 38 & $17^{\circ} 50^{\prime} 20.1^{\prime \prime}$ & $51^{\circ} 30^{\prime} 18.4^{\prime \prime}$ & 850 & Pequeno & Plantação & Sem vegetação \\
\hline 39 & $17^{\circ} 58^{\prime} 3.8^{\prime \prime}$ & $50^{\circ} 29^{\prime} 43.3^{\prime \prime}$ & 530 & Grande & Mata e pastagem & Arbustiva \\
\hline 40 & $17^{\circ} 48^{\prime} 24.3^{\prime \prime}$ & $51^{\circ} 47^{\prime} 28.9^{\prime \prime}$ & 630 & Médio & Mata e plantação & Herbácea, arbustiva e arbórea \\
\hline 41 & $17^{\circ} 08^{\prime} 49.8^{\prime \prime}$ & $52^{\circ} 40^{\prime} 6.9^{\prime \prime}$ & 600 & Pequeno & Mata & Herbácea, arbustiva e arbórea \\
\hline 42 & $18^{\circ} 15^{\prime} 21^{\prime \prime}$ & $52^{\circ} 54^{\prime} 31^{\prime \prime}$ & 790 & Grande & Mata & Herbácea, arbustiva e arbórea \\
\hline 43 & $18^{\circ} 15^{\prime} 30^{\prime \prime}$ & $52^{\circ} 53^{\prime} 22^{\prime \prime}$ & 760 & Grande & Mata & Herbácea, arbustiva e arbórea \\
\hline 44 & $17^{\circ} 40^{\prime} 26^{\prime \prime}$ & $50^{\circ} 59^{\prime} 46^{\prime \prime}$ & 775 & Médio & Mata & Herbácea, arbustiva e arbórea \\
\hline 45 & $15^{\circ} 55^{\prime} 25^{\prime \prime}$ & $51^{\circ} 43^{\prime} 25.7^{\prime \prime}$ & 625 & Pequeno & Plantação & Herbácea, arbustiva e arbórea \\
\hline
\end{tabular}

*Pequeno < $500 \mathrm{~m}^{2}$; Média: entre $500 \mathrm{~m}^{2}$ e $2000 \mathrm{~m}^{2}$; Grande $>2000 \mathrm{~m}^{2}$.

*Small < $500 \mathrm{~m}^{2}$; Average: entre $500 \mathrm{~m}^{2}$ e $2000 \mathrm{~m}^{2}$; Great > $2000 \mathrm{~m}^{2}$.

\section{Discussão}

Aproximadamente 150 espécies de anuros são conhecidas para o Cerrado (Bastos 2007). A riqueza de espécies encontradas na região sudoeste do Estado de Goiás corresponde à $25 \%$ das espécies do bioma e, apesar deste estudo cobrir uma área de amostragem superior a outras já amostradas neste bioma (Silveira 2006, Vaz-Silva et al 2007, Giaretta et al. 2008) a riqueza de espécies foi similar.
Segundo Diniz-Filho et al. (2005), existem ainda muitas regiões subamostradas no Cerrado, o que sugere que o número de espécies de anuros registrados até o momento seja subestimado. Nosso estudo corrobora esta observação, uma vez que registramos uma espécie ainda não conhecida formalmente pela ciência, Scinax sp. (aff. fuscomarginatus), que se em processo de descrição taxonômica (R. P. Bastos, dados não publicados). 

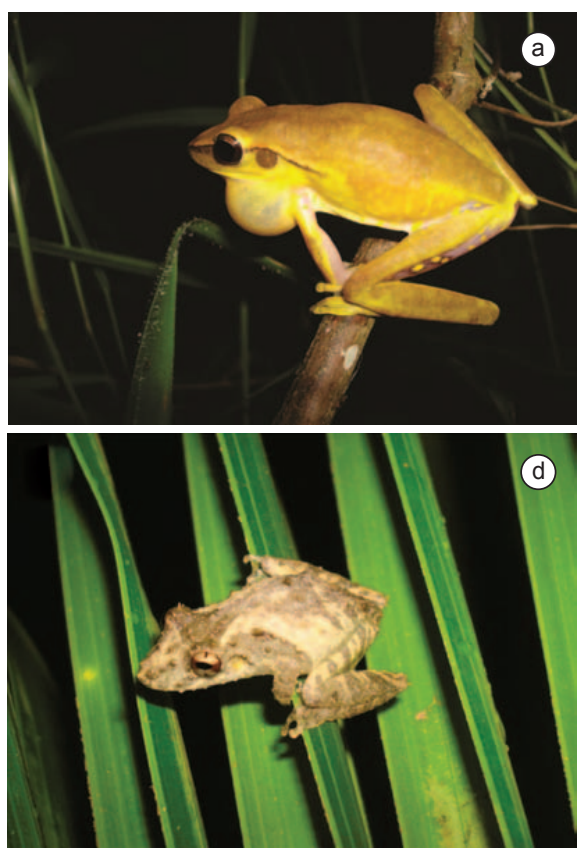

(d)
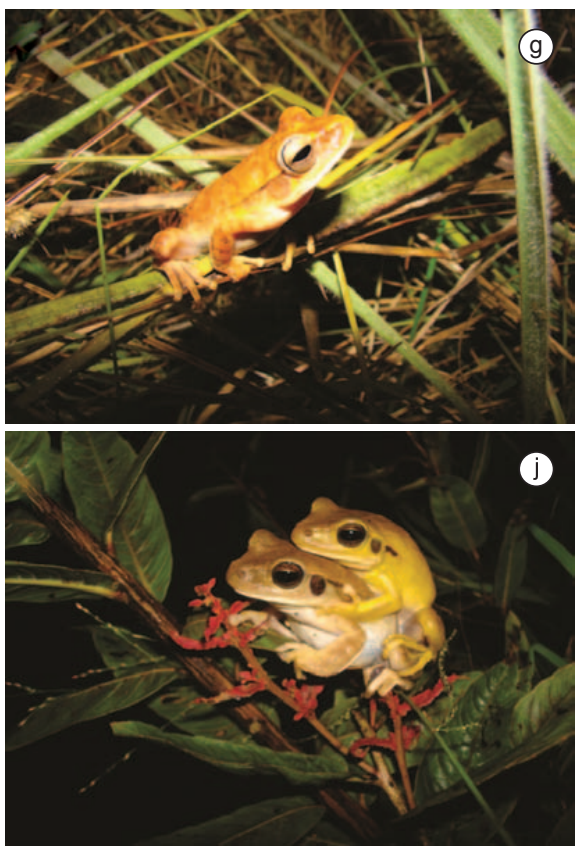
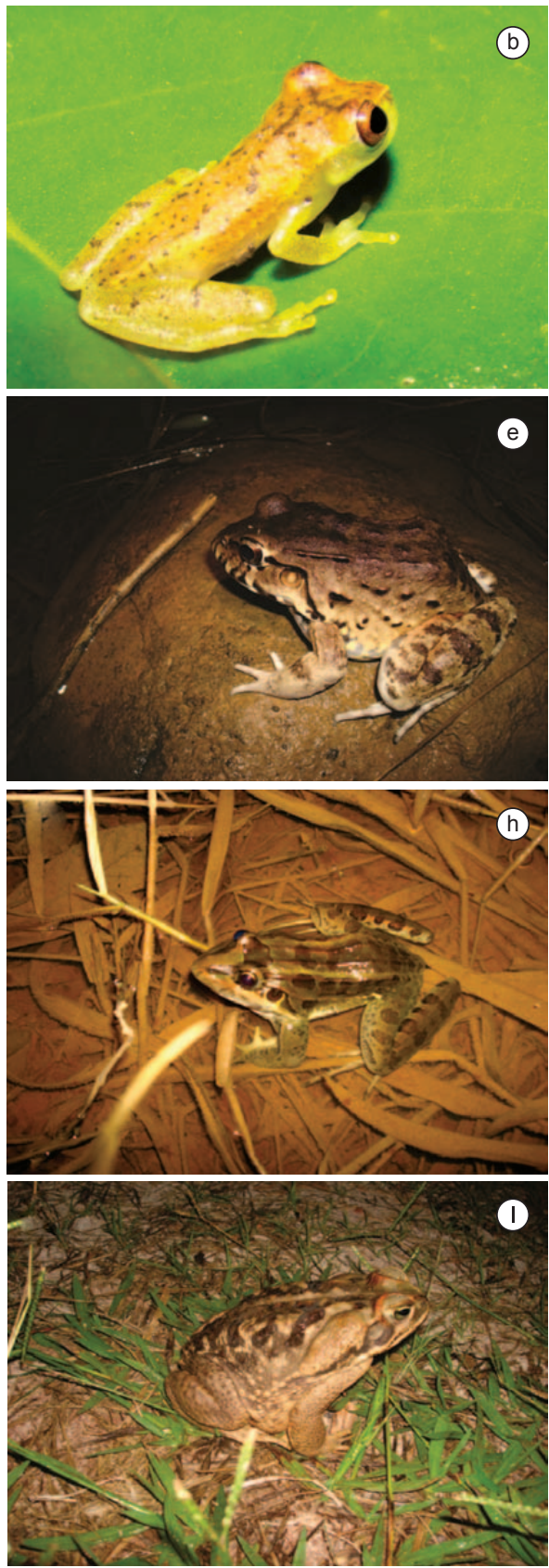
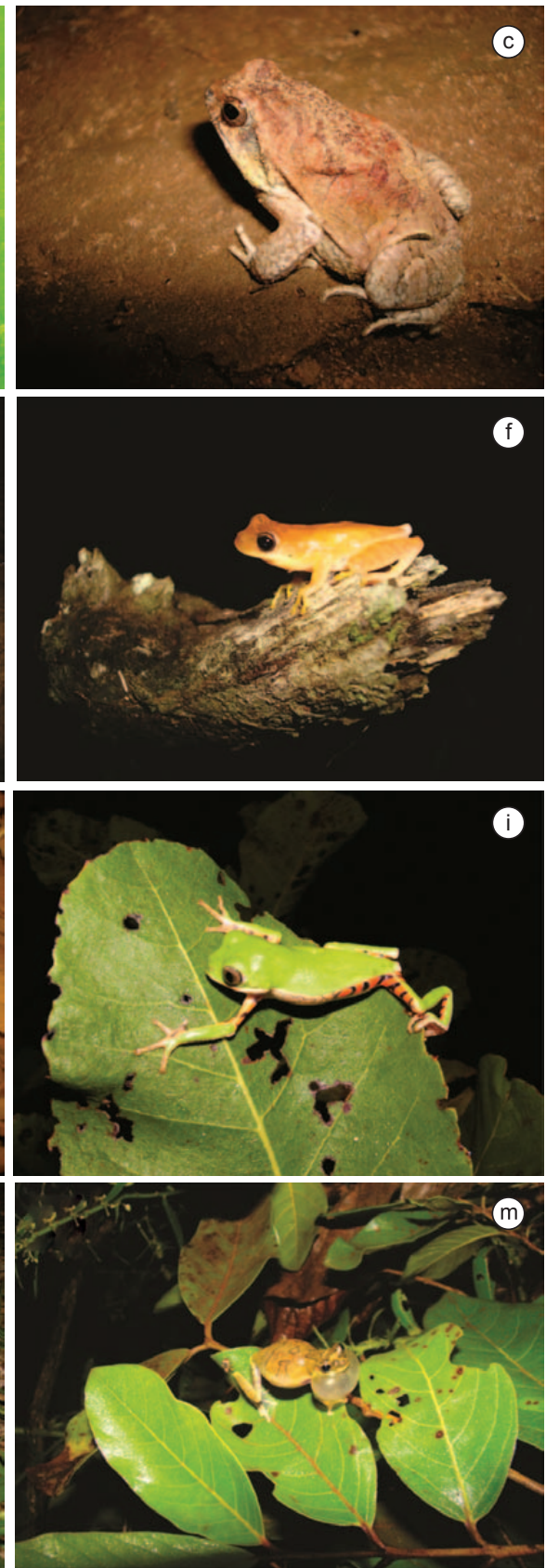

Figura 2. Espécies de anfíbios anuros encontradas na região sudoeste do estado de Goiás, Brasil. a) Hypsiboas albopunctatus; b) Dendropsophus cruzi c) Eupemphix nattereri; d); Scinax constrictus; e) Leptodactylus labyrinthicus; f) Dendropsophus minutus; g) Hypsiboas multifasciatus; h) Leptodactylus latrans; i) Phyllomedusa azurea; j) Hypsiboas raniceps; 1) Rhinella schneideri; m) Scinax fuscovarius.

Figure 2. Amphibian anuran species found in Southwest region of Goiás state, Brazil. a) Hypsiboas albopunctatus; b) Dendropsophus cruzi c) Eupemphix nattereri; d); Scinax constrictus; e) Leptodactylus labyrinthicus; f) Dendropsophus minutus; g) Hypsiboas multifasciatus; h) Leptodactylus latrans; i) Phyllomedusa azurea; j) Hypsiboas raniceps; 1) Rhinella schneideri; m) Scinax fuscovarius.

Tabela 2. Lista de espécies de anuros coletados no sudoeste do estado de Goiás, Brasil.

Table 2. List of anurans species collected in the Southwest of Goiás State, Brazil.

\begin{tabular}{|c|c|c|c|c|c|}
\hline \multirow[t]{3}{*}{ Família } & \multirow[t]{3}{*}{ Espécies } & \multicolumn{4}{|c|}{ Estação reprodutiva } \\
\hline & & \multicolumn{2}{|c|}{$2007 / 2008$} & \multicolumn{2}{|c|}{$2008 / 2009$} \\
\hline & & Adulto & Girino & Adulto & Girino \\
\hline \multirow[t]{2}{*}{ Bufonidae } & Rhinella mirandaribeiroi (Gallardo, 1965) & - & - & $\mathrm{X}$ & - \\
\hline & Rhinella schneideri (Werner, 1894) & $\mathrm{X}$ & - & $\mathrm{X}$ & $\mathrm{X}$ \\
\hline Cycloramphidae & Odontophrynus aff. americanus & - & - & $\mathrm{X}$ & - \\
\hline Hylidae & Dendropsophus cruzi (Pombal \& Bastos, 1998) & $\mathrm{X}$ & $\mathrm{X}$ & $\mathrm{X}$ & $\mathrm{X}$ \\
\hline
\end{tabular}


Tabela 2. Continuação...

\begin{tabular}{|c|c|c|c|c|c|}
\hline \multirow[t]{3}{*}{ Família } & \multirow[t]{3}{*}{ Espécies } & \multicolumn{4}{|c|}{ Estação reprodutiva } \\
\hline & & \multicolumn{2}{|c|}{$2007 / 2008$} & \multicolumn{2}{|c|}{$2008 / 2009$} \\
\hline & & Adulto & Girino & Adulto & Girino \\
\hline & Dendropsophus jimi (Napoli \& Caramaschi, 1999) & $\mathrm{X}$ & - & $\mathrm{X}$ & - \\
\hline & Dendropsophus minutus (Peters 1872) & $\mathrm{X}$ & $\mathrm{X}$ & $\mathrm{X}$ & $\mathrm{X}$ \\
\hline & Dendropsophus nanus (Boulenger, 1889) & $\mathrm{X}$ & $\mathrm{X}$ & $\mathrm{X}$ & $\mathrm{X}$ \\
\hline & $\begin{array}{l}\text { Dendropsophus rubicundulus ( Reinhardt and Lütken, } \\
\text { 1862) }\end{array}$ & $\mathrm{X}$ & $\mathrm{X}$ & $\mathrm{X}$ & $\mathrm{X}$ \\
\hline & Dendropsophus soaresi (Caramaschi \& Jim, 1983) & $\mathrm{X}$ & - & - & - \\
\hline & Hypsiboas albopunctatus ( Spix, 1824) & $\mathrm{X}$ & $\mathrm{X}$ & $\mathrm{X}$ & $\mathrm{X}$ \\
\hline & Hypsiboas lundii ( Burmeisteri, 1856) & $\mathrm{X}$ & $\mathrm{X}$ & $\mathrm{X}$ & $\mathrm{X}$ \\
\hline & Hypsiboas multifasciatus (Günther, 1859) & - & - & $\mathrm{X}$ & - \\
\hline & Hypsiboas raniceps Cope 1862 & $\mathrm{X}$ & $\mathrm{X}$ & $\mathrm{X}$ & $\mathrm{X}$ \\
\hline & Phyllomedusa azurea Cope, 1862 & $\mathrm{X}$ & $\mathrm{X}$ & $\mathrm{X}$ & - \\
\hline & Phyllomedusa sp. (gr. hypochondiralis) & - & - & - & $\mathrm{X}$ \\
\hline & Pseudis bolbodactyla Lutz, 1925 & - & - & $\mathrm{X}$ & $\mathrm{X}$ \\
\hline & Scinax gr. Ruber & $\mathrm{X}$ & $\mathrm{X}$ & - & - \\
\hline & Scinax constrictus Lima, Bastos \& Giaretta, 2004 & $\mathrm{X}$ & - & $\mathrm{X}$ & - \\
\hline & Scinax fuscomarginatus (Lutz, 1925) & $\mathrm{X}$ & $\mathrm{X}$ & $\mathrm{X}$ & $X$ \\
\hline & Scinax sp. (aff. fuscomarginatus) & $\mathrm{X}$ & - & $\mathrm{X}$ & $\mathrm{X}$ \\
\hline & Scinax fuscovarius (Lutz, 1925) & - & $\mathrm{X}$ & $\mathrm{X}$ & $\mathrm{X}$ \\
\hline & Scinax sp. 1 & - & $\mathrm{X}$ & - & - \\
\hline & Scinax sp. 2 & - & $\mathrm{X}$ & - & - \\
\hline & Scinax sp. 3 & - & $\mathrm{X}$ & - & - \\
\hline & Scinax $x$-signatus (Spix, 1824) & $\mathrm{X}$ & - & $\mathrm{X}$ & - \\
\hline \multirow[t]{7}{*}{ Leiuperidae } & Eupemphix nattereri (Steindachner, 1863) & $\mathrm{X}$ & $\mathrm{X}$ & $\mathrm{X}$ & $\mathrm{X}$ \\
\hline & Physalaemus centralis Bokermann, 1962 & $\mathrm{X}$ & - & $\mathrm{X}$ & $\mathrm{X}$ \\
\hline & Physalaemus cuvieri Fitzinger, 1826 & $\mathrm{X}$ & $\mathrm{X}$ & $\mathrm{X}$ & $\mathrm{X}$ \\
\hline & Physalaemus sp. & - & $\mathrm{X}$ & - & - \\
\hline & Pseudopaludicola sp. & - & - & - & $\mathrm{X}$ \\
\hline & Pseudopaludicola falcipes (Hensel, 1867) & - & - & $\mathrm{X}$ & - \\
\hline & Pseudopaludicola saltica (Cope, 1887) & $\mathrm{X}$ & - & $\mathrm{X}$ & - \\
\hline \multirow[t]{8}{*}{ Leptodactylidae } & Leptodactylus furnarius Sazima \& Bokermann, 1978 & $\mathrm{X}$ & $\mathrm{X}$ & $\mathrm{X}$ & $\mathrm{X}$ \\
\hline & Leptodactylus fuscus (Schneider, 1799) & $\mathrm{X}$ & $\mathrm{X}$ & $\mathrm{X}$ & $\mathrm{X}$ \\
\hline & Leptodactylus hylaedactylus (Cope, 1868) & - & - & $\mathrm{X}$ & - \\
\hline & Leptodactylus labyrinthicus (Spix, 1824) & $\mathrm{X}$ & $\mathrm{X}$ & $\mathrm{X}$ & $\mathrm{X}$ \\
\hline & Leptodactylus mystacinus (Spix, 1824) & - & - & $\mathrm{X}$ & - \\
\hline & Leptodactylus latrans (Linnaeus, 1758) & $\mathrm{X}$ & - & $\mathrm{X}$ & - \\
\hline & Leptodactylus podicipinus (Cope, 1862) & - & - & $\mathrm{X}$ & - \\
\hline & Leptodactylus syphax Bokermann, 1969 & - & - & $\mathrm{X}$ & - \\
\hline Microhylidae & Elachistocleis ovalis (Schneider, 1799) & $\mathrm{X}$ & $\mathrm{X}$ & $\mathrm{X}$ & $\mathrm{X}$ \\
\hline Riqueza de espécies & & 25 & 21 & 33 & 21 \\
\hline
\end{tabular}

Quanto à curva de acumulação de espécies, tanto para a fase adulta quanto para a larval, foi verificada uma tendência à estabilização. Desta forma, verifica-se que o esforço amostral ou técnica de coleta foi eficiente na amostragem das espécies presentes na área. Todavia, como os girinos permanecem na poça por um período de tempo maior, seria esperado que o número de espécies de anuros na fase larval fosse superior ao de adultos, como argumentado por Lips \& Savage (1996), Altig \& McDiarmid (1999) e Rossa-Feres \& Nomura (2006). $\mathrm{Na}$ área amostrada, o menor número de registros na fase larvária parece ser decorrente da dificuldade de coleta dos girinos em corpos d'água muito grandes e profundos, o que limita a amostragem apenas à porção marginal.

A maioria das espécies registradas apresenta ampla distribuição, ocorrendo em mais de um bioma brasileiro ou até em países vizinhos (Frost 2010). Somente Hypsiboas lundii, Scinax sp. (aff. fuscomarginatus) e Scinax constrictus apresentam distribuição restrita ao Brasil Central (Frost 2010, R. P. Bastos, dados não publicados). Segundo Duellman (1999) ocorre nas regiões neotropicais uma predominância das famílias Hylidae e Leptodactylidae, assim para a região sudoeste de Goiás foi observado um maior número de 

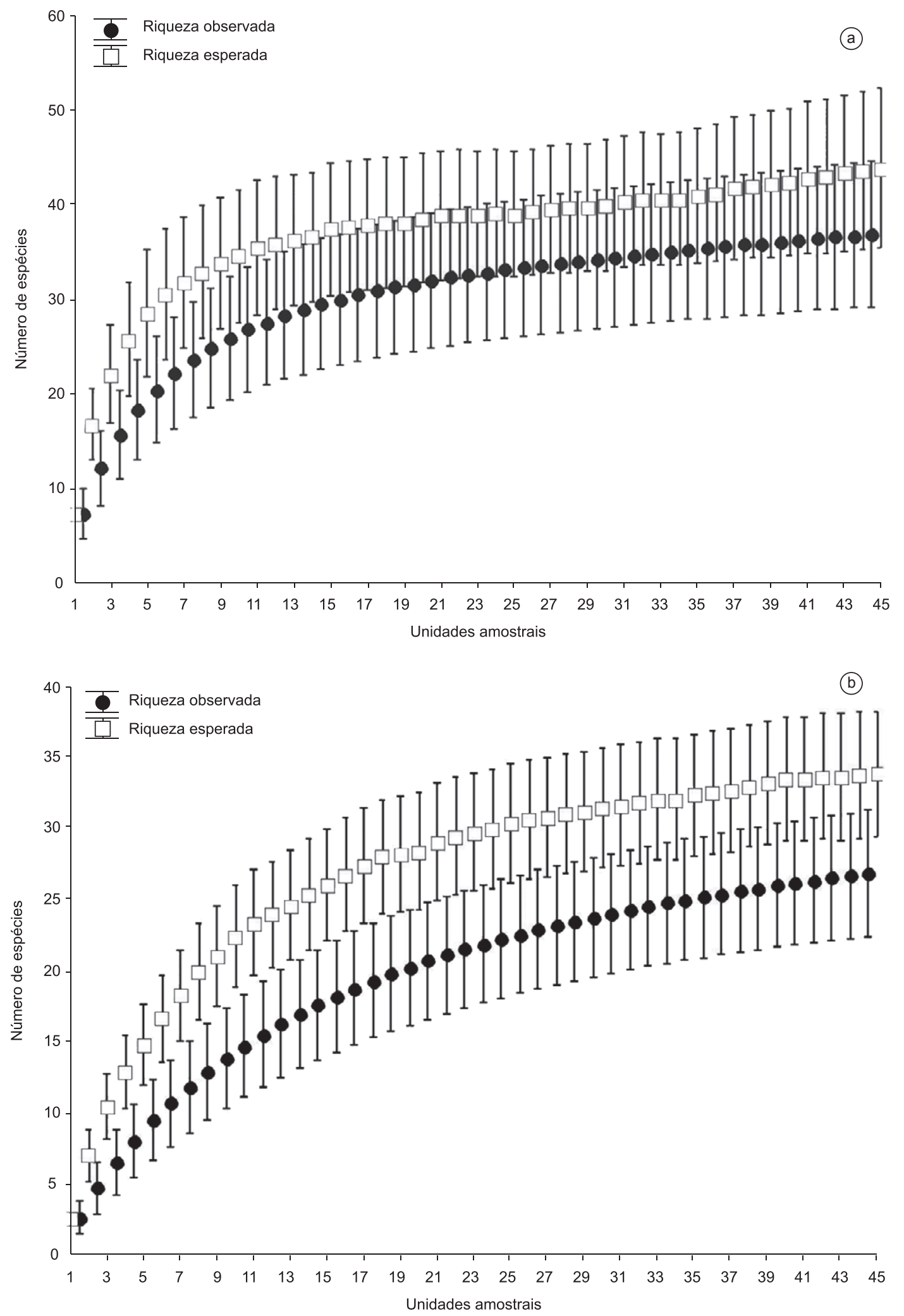

Figura 3. a) Curva de acumulação de espécies (riqueza observada e estimada - Jackknife 1) de espécies de anfíbios anuros (em fase adulta), sudoeste do estado de Goiás, Brasil; b) Curva de acumulação de espécies (riqueza observada e estimada - Jackknife 1) de espécies de anfíbios anuros (em fase larval), sudoeste do estado de Goiás, Brasil.

Figure 3. a) Rarefied curve of amphibian anuran species richness (observed and estimated - Jackknife 1) (adult), southwest of Goias state, Brazil; b) Rarefied curve of amphibian anuran species richness (observed and estimated - Jackknife 1) (tadpole), southwest of Goias state, Brazil. 
espécies para estas famílias, semelhante ao encontrado em outras áreas de Cerrado (Bastos et al. 2003, Brasileiro et al. 2005, Silveira 2006, Vaz-Silva et al. 2007).

Grande parte das espécies registradas se reproduz em áreas abertas, não apresentando grande especificidade de sítios reprodutivos, portanto podendo ser consideradas habitat-generalistas (Brasileiro et al. 2005). Porém, espécies como H. lundii e Leptodactylus syphax demonstraram especificidade de habitat, ocorrendo em ambientes florestais ou de afloramento rochoso, respectivamente, como já registrado em outros estudos (Bastos et al. 2003, Silva \& Giaretta 2009).

Suazo-Ortuno et al. (2007) e Silva et al. (2009) afirmam que paisagens de agrossistemas podem influenciar negativamente as comunidades de anuros, seja diminuindo a riqueza de espécies ou favorecendo a ocorrência daquelas mais generalistas, com tais mudanças ocorrendo em virtude das alterações na cobertura vegetal dos habitats. Neste estudo, apesar da maioria dos corpos d'água estarem envoltos principalmente por grandes plantações e pastagens, observamos uma alta riqueza de espécies, que pode ser comparável a grande diversidade de espécies encontradas em áreas protegidas neste bioma (Eterovick \& Sazima 2004, Uetanabaro et al. 2007). Entretanto, esta semelhança no número de espécies deve-se provavelmente à diferença de tamanho entre as áreas amostradas, uma vez que a área amostrada no sudoeste goiano (este estudo) é maior.

Todavia em anfíbios, medidas simples de diversidade, tais como riqueza de espécie, podem não refletir adequadamente a perda real da diversidade ocasionada por distúrbios antrópicos (Ernst et al. 2006), sendo necessária a utilização de outras métricas, por exemplo: diversidade filogenética (Magurran 2004) e/ou funcional (Tilman 2001), para que seja possível mensurar tal impacto. Desta forma, alterações nos habitats podem não influenciar negativamente o número de espécies em uma comunidade, mas sim a identidade das mesmas, podendo acarretar em uma redução nos valores destas formas de diversidade.

\section{Agradecimentos}

Agradecemos aos dois revisores anônimos e à Dr $^{\mathrm{a}}$ Denise de Cerqueira Rossa-Feres pela leitura critica e sugestões ao trabalho. Somos gratos ao Instituto Chico Mendes de Conservação da Biodiversidade (ICMbio) pela licença concedida; RPB agradece ao CNPQ pelo auxílio concedido. Os autores são gratos a FAPEG, CNPQ e FUNAPE/UFG pelo apoio financeiro.

\section{Referências Bibliográficas}

ALCÂNTARA, M.B., LIMA, L.P. \& BASTOS, R.P. 2007. Breeding activity of Scinax centralis (Anura, Hylidae) in Central Brazil. Iheringia, Ser. Zool. 97(4):406-410.

ALTIG, R. \& McDIARMID, R.W. 1999. Diversity: Familial and Generic Characterizations. In Tadpoles. The Biology of Anuran Larvae (R.W. McDiarmid \& R. Altig, eds). University of Chicago Press, Chicago and London, p.295-337.

BASTOS, R.P. 2007. Anfíbios do Cerrado. In Herpetologia no Brasil II (L.B. Nascimento \& M.E., Oliveira, eds). Sociedade Brasileira de Herpetologia. Belo Horizonte, p.87-100.

BASTOS, R.P., MOTTA, J.A.O., LIMA, L.P. \& GUIMARÃES, L.D. 2003. Anfíbios da Floresta Nacional de Silvânia, estado de Goiás. Goiânia.

BRANDÃO, R.A. \& ARAÚJO, A.F.B. 1998. A herpetofauna da estação ecológica Águas Emendadas. In Vertebrados da estação ecológica Águas Emendadas, história natural e ecologia em um fragmento de cerrado do Brasil Central (J. Marinho-Filho, J., Rodrigues \& M. Guimarães, eds). SEMATEC/IEMA, Brasília, p.9-21.

BRANDÃO, R.A. 2002. A new species of Phyllomedusa Wagler, 1830 (Anura: Hylidae) from Central Brazil. J. Herpetol. 36(4):571-578.
BRASILEIRO, C.A., SAWAYA, R.J., KIEFER, M.C. \& MARTINS, M. 2005. Amphibians of the Cerrado of Itirapina Ecological Station, Southeastern Brazil. Biota Neotrop. 5(2):1-17.

CODDINGTON, J.A., GRISWOLD, C.E., DÁVILA, D.S., PEÑARANDA, E. \& LARCHER, S.F. 1991. Designing and testing sampling protocols to estimate biodiversity in tropical ecosystems. In The unity of evolutionary biology: proceedings of the Fourth International Congress of Systematic and Evolutionary Biology (E. C. Dudley, ed.). Dioscorides Press, Portland, p.44-60.

COLLI, G.R., BASTOS, R.P. \& ARAÚJO A.F.B. 2002. The character and dynamics of the Cerrado Herpetofauna. In The Cerrados of Brazil: Ecology and Natural History of a Neotropical Savanna (P. S. Oliveira \& R.J. Marquis, eds). Columbia University Press, New York, p.223-241.

COLWELL, R.K. \& CODDINGTON, J.A. 1994. Estimating terrestrial biodiversity through extrapolation. Philos. T. R. Soc. B. 345:101-118. PMid:7972351. http://dx.doi.org/10.1098/rstb.1994.0091

CRUMP, M. \& SCOTT JUNIOR, N.J. 1994. Standard techniques for Inventory and Monitoring. In Measuring and Monitoring Biological Diversity (W.R. Heyer, M.A. Donnelly, R.W. McDiarmid, L.A.C. Hayek \& M.S. Foster, eds). Smithsonian Institutions Press, Washington and London, p.84-92.

DINIZ-FILHO, J.A.F., BINI, L.M., VIEIRA, C.M., SOUSA, M.C., BASTOS, R.P., BRANDÃO, D., OLIVEIRA, L.G. 2004a. Spatial patterns in species richness and priority áreas for conservation of anurans in the Cerrado region, central Brazil. Amphibia-Reptilia 25:63-75. http://dx.doi. org/10.1163/156853804322992841

DINIZ-FILHO, J.A.F., BINI, L.M., BASTOS, R.P., VIEIRA, C.M., SOUSA, M.C., MOTTA, J.A.O., POMBAL JUNIOR, J.P. \& PEIXOTO, J.C. 2004b. Anurans from a local assemblage in Central Brazil: Liking local processes with macroecological patterns. Braz. J. Biol. 64(1):41-52. http://dx.doi.org/10.1590/S1519-69842004000100006

DINIZ-FILHO, J.A.F., BASTOS, R.P., RANGEL,T.F.L.V.B., BINI, L.M., CARVALHO, P. \& SILVA, R.J. 2005. Macroecological correlates and spatial patterns of anuran description dates in the Brazilian Cerrado. Global Ecol Biogeogr. 14:469-477. http://dx.doi.org/10.1111/j.1466822X.2005.00165.X

DINIZ-FILHO, J.A.F., BINI, L.M., OLIVEIRA, G., BARRETO, B.S., SILVA, M.M.F.P., TERRIBLE, L., RANGEL, T.F.L.V., PINTO, M.P., SOUSA, N.P.R., VIEIRA, L.C.G., MELO, A.S., DE MARCO JUNIOR, P., BLAMIRES, D., BASTOS, R.P., CARVALHO, P., FERREIRA, L.G., TELLES, M.P.C., RODRIGUES, F.M., SILVA, D.M. SILVA JUNIOR, N.J. \& SOARES, T.N. 2009. Macroecologia, biogeografia e áreas prioritárias para conservação no cerrado. Oecol. Bras. 13:470-497. http://dx.doi.org/10.4257/oeco.2009.1303.05

DUELLMAN, W.E. 1999. Distribution patterns of amphibians in South American. In Patterns of distribution of amphibians: a global perspective (W.E. Duellman, ed). The Johns Hopkins University Press, Baltimore, p.255-481.

ERNST, R., LINSENMAIR, K.E. \& RÖDEL, M.O. 2006. Diversity erosion beyond the species level: Dramatic loss of functional diversity after selective logging in two tropical amphibian communities. Biological Conservation 133:143-155. http://dx.doi.org/10.1016/j. biocon.2006.05.028

ETEROVICK, P.C. \& SAZIMA, I. 2000. Structure of an anuran community in a montane meadow in southeastern Brazil: effects of seasonality, habitat and predation. Amphibia-Reptilia 21:439-461. http://dx.doi. org/10.1163/156853800300059331

ETEROVICK, P.C. \& SAZIMA, I. 2004. Anfíbios da Serra do Cipó, Minas Gerais. Editora PUC Minas, Belo Horizonte.

FROST, D.R. 2010. Amphibian Species of the world: an online reference. Version 5.4. American Museum of Natural History, New York. http://research.amnh.org/vz/herpetology/amphibia (último acesso em 16/12/2010).

GIARETTA, A.A., MENIN, M., FACURE, K.G., KOKUBUM, M.N.C. \& OLIVEIRA-FILHO, J.C. 2008. Species richness, relative abundance, and habitat of reproduction of terrestrial frogs in the Triângulo Mineiro region, Cerrado biome, southeastern Brazil. Iheringia. Ser Zool. 98(2):181-188. 
GUIMARÃES , L.D. \& BASTOS, R.P. 2003. Vocalizações e Interações acústicas em Hyla raniceps (Anura, Hylidae) durante a atividade reprodutiva. Iheringia. Ser Zool. 93(2):149-158.

HADDAD, C.F.B. 2008. Uma análise da lista brasileira de anfíbios ameaçados de extinção. In Livro Vermelho da Fauna Brasileira Ameaçada de Extinção (Machado, A. B. M.; Drummond, G. M. and A. P. Paglia, eds.). MMA e Fundação Biodiversitas, Brasília e Belo Horizonte, p.286-325.

HEYER, W.R., DONNELLY, M.A., MCDIAMIRD, R.W., HAYEK, L.C. \& FOSTER, M. 1994. Measuaring and monitoring biological diversity standard methods for amphibians. Smithsonian Institutions Press, Washington, D.C.

INTERNATIONAL UNION FOR CONSERVATION OF NATURE - IUCN. 2010. IUCN Red List of Threatened Species. Version $2010.1 \mathrm{http}: / /$ www. iucnredlist.org (ultimo acesso em 28/12/2010).

KLINK, C.A. \& MACHADO, R. 2005. Conservation of the Brazilian Cerrado. Conserv. Biol., 19:707-713.

KREBS, C.J. 1999. Ecological Methodology, Addison Wesley Longman, Inc., Menlo Park.

LIPS, K.R. \& SAVAGE, J.M. 1996. Key to know tadpoles (Amphibia: Anura) of Costa Rica. Stud. Neotrop. Fauna Environ. 31:17-26. http://dx.doi. org/10.1076/snfe.31.1.17.13320

MAGURRAN, A.E. 2004. Measuring biological diversity. Blackwell, Oxford.

MARTINS, I.A. \& JIM, J. 2003. Bioacustic analysis of adverstisement call in Hyla nana and H. sanborni (Anura: Hylidae) in Botucatu, São Paulo, Brasil. Braz. J. Biol. 63(3):507-516. http://dx.doi.org/10.1590/S151969842003000300017

MINER, E. 1989. Climatologia do Brasil. Instituto Brasileiro de Geografia e Estatística - IBGE, Rio de Janeiro.

MITTERMEIER, R.A., MYERS, N., THOMSEN, J.B., FONSECA, G.A.B \& OLIVEIRA, S. 1998. Biodiversity hotspots and major tropical wilderness areas: approaches to setting conservation priorities. Conserv. Biol. 12(3):516-520.

MYERS, N., MITTERMEIER, R.A., MITTERMEIER, C.G., FONSECA, G.A.B. \& KENT, J. 2000. Biodiversity hotspots for conservation priorities. Nature 403(24):853-858. PMid:10706275. http://dx.doi. org/10.1038/35002501

PUGLIESI, A., ALVES, A.C. \& POMBAL JUNIOR, J.P. 2001. The tadpole of Hyla rubicundula (Anura: Hylidae). J. Herpetol. 35(4):686-688. http:// dx.doi.org/10.2307/1565914

ROSSA-FERES, D.C. \& JIM, J. 2001. Similaridade do sítio de vocalização em uma comunidade de anfíbios anuros na região noroeste do Estado de São Paulo, Brasil. Rev. Bras. Zool. 18(2):439-454. http://dx.doi.org/10.1590/ S0101-81752001000200015
ROSSA-FERES, D.C. \& NOMURA, F. 2006. Characterization and taxonomic key for tadpoles (Amphibia: Anura) from the northwestern region of São Paulo State, Brazil. Biota Neotrop. 6(1): http://www.biotaneotropica.org. br/v6n1/pt/abstract?identification-key+bn00706012996 (último acesso em 12/10/2010).

SANTOS, A.J. 2006. Estimativas de riqueza em espécies. In Métodos de estudo em Biologia da Conservação \& Manejo da Vida Silvestre (L. Cullen, R. Rudran, \& C. Valladares-Padua, eds). Editora da Universidade Federal do Paraná, Curitiba, p.19-43.

SILVA, F.R., SANTOS, R.S., NUNES, M.A. \& ROSSA-FERES, D. C. 2009. Anuran captured in pitfall traps in three agricultural cultivations. Biota Neotrop. 9(4):253 -255 http://www.biotaneotropica.org.br/v9n4/ en/abstract?short-communication+bn01109042009 (último acesso em 15/10/2010).

SILVA, W.R. \& GIARETTA, A.A. 2009. On the natural history of Leptodactylus syphax with comments on the evolution of reproductive features in the $L$. pentadactylus species group (Anura, Leptodactylidae). Journal of Natura History 43(3):191-203. http://dx.doi.org/10.1080/00222930802484618

SILVEIRA, A.L. 2006. Anfíbios do Município de João Pinheiro, uma área de Cerrado no noroeste de Minas Gerais, Brasil. Arq. Mus. Nac. 64:131-139.

SUAZO-ORTUNO, I., DIAZ, J.A. \& MARTINEZ-RAMOS, M. 2007. Effects of Conversion of Dry Tropical Forest to Agricultural Mosaic on Herpetofaunal Assemblages. Conserv. Biol. 22(2):362-374. http://dx.doi. org/10.1111/j.1523-1739.2008.00883.x

TILMAN, D. 2001. Functional diversity. In Encyclopedia of Biodiversity (S.A. Levin, ed). Academic Press, San Diego, p.109-120. http://dx.doi. org/10.1016/B0-12-226865-2/00132-2

UETANABARO, M., SOUZA, F.L., LANDGREF-FILHO, P., BEDA, A.F. \& BRANDÃO, R.A. 2007. Anfíbios e répteis do Parque Nacional da Serra da Bodoquena, Mato Grosso do Sul, Brasil. Biota Neotrop 7(3):279290. http://www.biotaneotropica.org.br/v7n3/pt/abstract?inventory+ bn01207032007 (ultimo acesso em 10/07/2011).

VALENTE, C.R. 2006. Caracterização Geral e Composição Florística do Cerrado. In Natureza Viva Cerrado, caracterização e conservação (L.D. Guimarães, M.A.D. Silva \& T.C. Anacleto, eds). Editora UCG, Goiânia, p.19-54.

VAZ-SILVA, W., GUEDES, A.G., SILVA, P. L.A., GONTIJO, F.F., BARBOSA, R.S., ALOISIO, G.R. \& OLIVEIRA, F.C.G. 2007. Herpetofauna, Espora Hydroelectric Power Plant, state of Goiás, Brazil. Check List 3:338-345.

WALTHER, B.A. \& MORAND, S. 1998. Comparative performance of species richness estimation methods. Parasitology 116:395-405. PMid:9585941. http://dx.doi.org/10.1017/S0031182097002230

ZIMMERMAN, B.L. 1994. Audio strip transects. In Measuring and Monitoring Biological Diversity (W.R. Heyer, M.A. Donnelly, R.W. McDiarmid, L.A.C. Hayek \& M.S. Foster, eds.). Smithsonian Institutions Press, Washington and London, p.92-97. 